Perspective

\title{
Molecular sieve membranes: From 3D zeolites to 2D MOFs
}

\author{
Yanshuo Li*, Weishen Yang
}

\section{Introduction}

Gas separation with membranes is an energy efficient and environmentally friendly alternative to cryogenic, adsorptive or absorptive gas separation processes. Moreover, membranes units can be integrated easily into other separation and reaction processes. Nowadays, several applications based on polymeric membranes have achieved commercial success, e.g., $\mathrm{N}_{2}$ production from air, $\mathrm{H}_{2}$ recovery from ammonia purge gas, $\mathrm{CO}_{2}$ removal from natural gas and $\mathrm{CO}_{2}$ separation from hydrocarbons for enhanced oil recovery (EOR). Traditional polymeric membranes that rely on a solution-diffusion separation mechanism are subject to a tradeoff between productivity (permeability) and efficiency (selectivity), which is known as Robeson's upper bound [1]. Membranes based on molecular sieve materials can overcome this limitation because of their ability to separate molecules based on size and shape [2-4].

\section{Achievements in zeolite membranes}

A zeolite is an inorganic molecular sieve. Unlike amorphous carbonaceous molecular sieves, crystalline zeolites contain uniformly sized pores of molecular dimensions, which makes them ideal candidates for molecular sieve membranes. The last two decades have witnessed continuous progress in the synthesis of zeolite membranes. Several strategies and techniques have been developed for the synthesis of zeolite membranes, including in-situ crystallization [5] and secondary (seeded) growth [6]. Significant improvements in membrane performance have been achieved by the microstructure optimization of zeolite membranes. For example, Tsapatsis et al. [2] demonstrated the amazing ability of zeolite membranes for separating molecules with extreme precision. A channel-orientation-optimized siliceous ZSM-5 membrane showed superior performance for the separation of $p$-xylene from its isomers ( $o$-xylene and $m$-xylene). This separation is very difficult because $p$-xylene is only $0.06 \mathrm{~nm}$ larger than the other isomers.

Despite impressive academic achievements, the commercial application of zeolite membranes is still limited to small-medium scale organic dewatering processes based on the hydrophilic LTA zeolite membrane, after its inception in 1999 as a semi-commercial installation in Japan by Mitsui Engineering and Shipbuilding Co. Ltd. [7]. The full scale commercialization is hampered by insufficient performance, high cost and difficulties in scale-up synthesis. In recent years, researchers at Dalian Institute of Chemical Physics (DICP) have devoted intense research efforts to the microwave synthesis of zeolite membranes [5,6,8-10]. In many cases, microwave synthesis remarkably reduced the synthesis time. In addition, microwave synthesis can also substantially eliminate the formation of non-zeolitic pores as compared with those synthesized by conventional heating [11]. Moreover, fast and volumetric microwave heating provides a promising solution to many problems encountered in large scale membrane production, such as thermal gradients and density gradients in the synthesis autoclaves. In 2012, Dalian HST Co. Ltd., a start-up company based on the DICP's technology, installed an LTA zeolite membrane unit for isopropanol dewatering with the capacity of 50,000 ton/year. The separation unit consists of 35 modules with a total permeation area of $350 \mathrm{~m}^{2}$, which is by far the largest zeolite membrane facility in the world (Fig. 1).

\section{Emerging of MOF membranes}

MOFs are novel crystalline hybrid materials consisting of metal ions or clusters interconnected by a variety of organic linkers [12-14]. In addition to promising applications in gas storage, adsorptive gas separation, functional devices and catalysis, their unique properties such as highly diversified structures, high surface areas and specific adsorption affinities make MOFs excellent candidates for use as molecular sieve membranes with superior performances. The preparation of gas separating MOF membranes is rapidly becoming a research focus in the current membrane community [15-21].

Synthetic techniques that have been developed and verified for zeolite membranes can be readily transferred to the preparation of MOF membranes. For example, microwave synthesis has been successful in the in-situ synthesis of ZIF-8 membrane (Fig. 2(a)) [15] and secondary growth of ZIF-7 membranes (Fig. 2(b)) [16], respectively. The concept of channel-orientationmanipulation has also been used for the microstructure optimization of MOF membranes. A c-out-of-plane oriented ZIF-7 


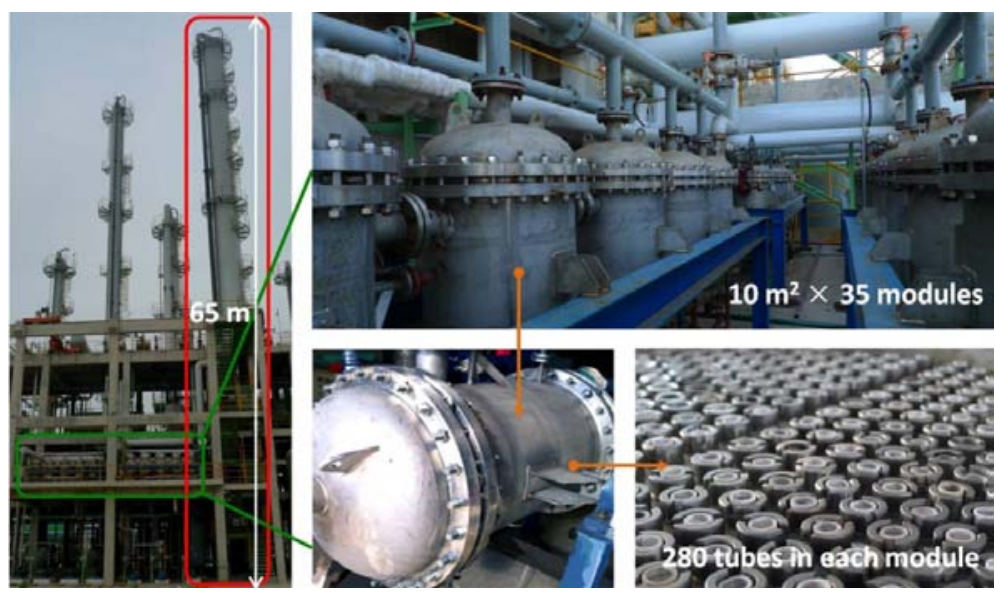

Fig. 1. LTA zeolite membrane unit (50000 ton/year) for isopropanol dewatering. The membrane unit (in green frame) replaces the dewatering column (in red frame), achieving a remarkable reduction in energy consumption.

\section{(a)}
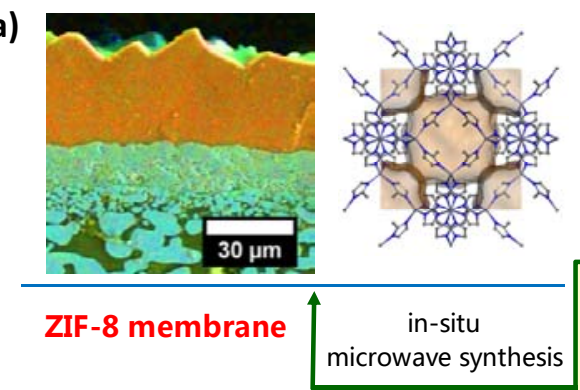

microwave synthesis

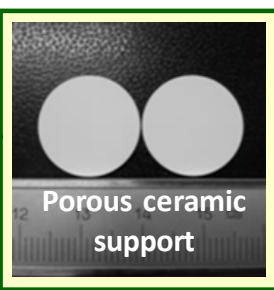

(c)
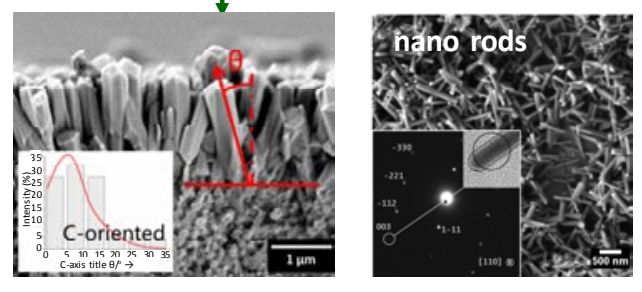

c-out-of-plane ZIF-7 membrane (b)

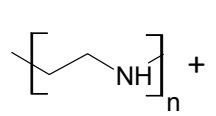

nano seeds + PEI

microwave-assisted secondary growth

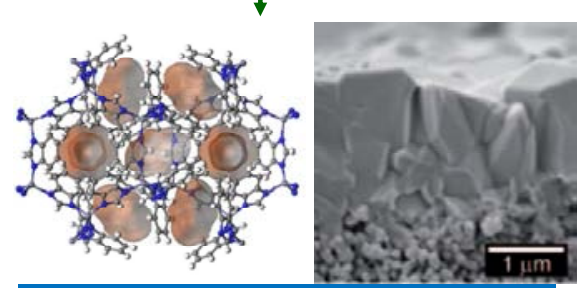

ZIF-7 membrane

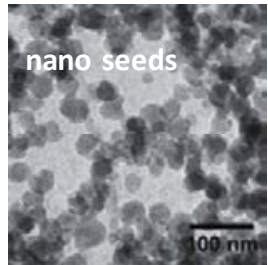

ane

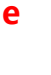

Fig. 2. Synthesis of MOF membranes with different methods. (a) in-situ microwave synthesis of ZIF-8 membrane on titanium support (reproduced from Ref. [15] by permission of the American Chemical Society); (b) microwave-assisted secondary growth of ZIF-7 membrane on aluminum support (reproduced from Ref. [16] by permission of John Wiley \& Sons Ltd.); (c) secondary growth of $c$-out-of-plane ZIF-7 membrane through evolutionary selection (reproduced from Ref. [17] by permission of John Wiley \& Sons Ltd.).

membrane was obtained by evolutionary selection in a van der Drift's type growth originating from randomly oriented seed layers by the altering of the relative growth rates of different crystal faces (Fig. 2(c)) [17]. The same method has also been employed for the synthesis of $<110>$ oriented ZIF-8 membranes [18].

MOF membranes are expected to bring forth a revolution in the field of membrane gas separation. In the last five years, several types of MOF membranes including ZIFs (Zeolitic Imidazolate Frameworks) [15-18], IRMOFs (Isoreticular MOFs) [19], MIL (Matériaux de l'Institut Lavoisier) series [20] and CAU (Christian Albrechts University) series [21] have been prepared and evaluated for various gas separation. However, in most cases, their separation selectivity was less satisfactory than that predicted by computer simulation, as shown in Fig. 3(a) [22]. It is generally accepted that the framework flexibility of MOFs can account for this. Taking ZIF-7 as an example as shown in Fig. 3(b), the low energy terahertz vibration in ZIF-7 and the flexiblity of the pore architecture, which were recently unambiguously identified by Tan et al. [23], explained the absence of a clear cut-off that was expected from the pore size estimated from the rigid framework models.

The framework flexibility of MOFs, on the other hand, is beneficial when the elasticity of MOF materials is favourable, 
(a)

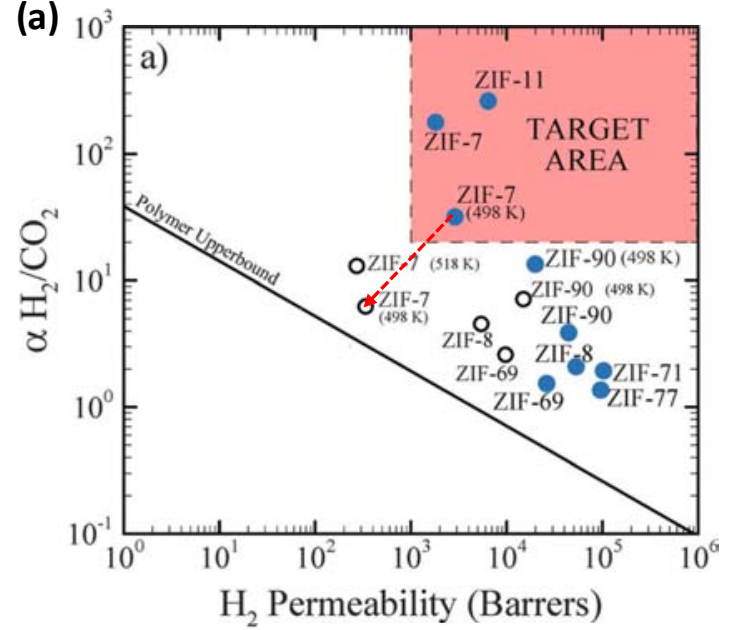

(b)

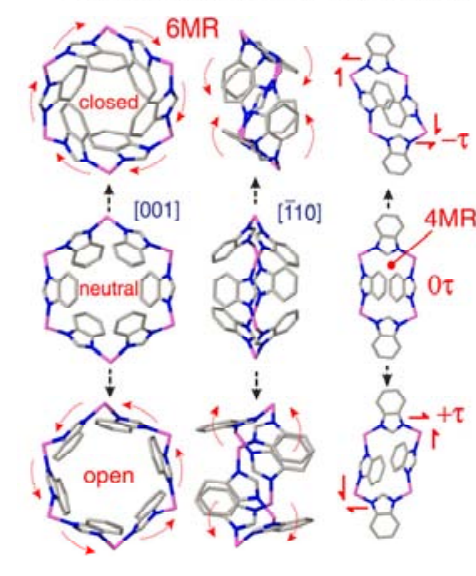

Breathing at $1.47 \mathrm{THz}$

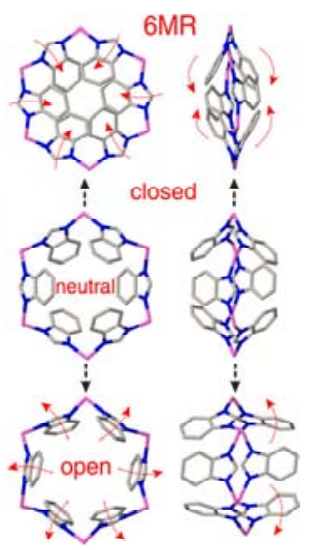

Fig. 3. (a) Predicted $\mathrm{H}_{2} / \mathrm{CO}_{2}$ selectivity (filled symbols) and experimental results (empty symbols) for ZIF membranes (reproduced from Ref. [22] by permission of John Wiley \& Sons Ltd.); (b) gate-opening and breathing motions of ZIF-7 identified at 0.654 and 1.47 THz. The first is a twisting spiral motion of benzimidazole linkers, and the second is a conventional gate-opening mechanism via synchronous flapping of benzimidazole linkers (reproduced from Ref. [23] by permission of the American Society of Physics).

e.g. in adsorption based separation applications, because the flexible materials can give high selectivity for guest inclusion by adapting the framework structure accordingly. For example, ZIF-8 nanoparticles have exhibited exceptional adsorption selectivity and capacity toward $i$-butanol molecules, showing a reversible gate-opening effect with the variation of $i$-butanol pressure or temperature [24]. Based on this observation, researchers at DICP incorporated ZIF-8 nanoparticles in a silicone rubber (polymethylphenylsiloxane, PMPS) matrix to fabricate organophilic pervaporative membranes. The ZIF-8-PMPS membrane showed very promising performance for recovering bio-alcohols from dilute aqueous solution [24].

\section{2D molecular sieve membranes}

It has long been a dream to make an artificial membrane with $\mathrm{nm}$ thickness and well-defined pores that can rapidly filter molecules with high resolution. In this regard, two dimensional (2D) molecular sieve nanosheets (MSNs) are the most appropriate building blocks for this kind of membranes. In recent years, ambitious attempts have been made to prepare MSNs and MSN membranes. However, progress has been limited [25].

Zeolite nanosheets or nanoplates can be synthesized by a bottom-up strategy as shown by Valtchev and Mintova [26]. However, the lateral size of the EMT zeolite nanosheets obtained by this method was only several nm, which is not suitable for use as building blocks for MSN membranes. The idea of using exfoliated zeolite nanosheets, a top-down strategy, was first proposed by Tspatsais et al. [27] in 2003. After ten years of continuous effort, they successfully developed a novel exfoliation method based on melt blending with a polymer, which ensured the structure preservation of the exfoliated zeolite layers including the MFI and MWW structural types [28]. They further solved the problem of the removal of the polymer and purification of the 2D zeolites from the non-exfoliated particles by gradient centrifugation [29]. After the filtration of the sus- pension of 2D zeolite nanosheets through a porous support and a gentle secondary growth, a molecular sieve membrane was obtained with a thickness of $100 \mathrm{~nm}$, which was 10 times thinner than typical 3-dimensional (3D) zeolite membranes [29]. This was an important breakthrough in the development of MSN membranes. Nevertheless, only 13 layered or 2D zeolite forms have been recognized so far [30], which significantly hinders research on zeolite-based MSN membranes.

Layered MOFs materials with abundant porous structure and functional surface groups can serve as a diverse source of MOF-based MSN membranes [31-34]. However, structural deterioration and morphological damage during the preparation have hampered success in obtaining high quality MOF nanosheets, which significantly hindered their application as building blocks for molecular sieve membranes. Recently, two major breakthroughs on the synthesis of morphology and structure preserving MOF nanosheets were achieved by the researchers from DICP and Delft University of Technology. The former developed a top down strategy, namely, a 2D MOF,

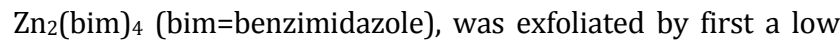
energy wet ball milling followed by exfoliation in a solvent under ultrasonication [35]. As shown in Fig. 4(a), effective exfoliation with the structural and morphological integrity of the in-plane structure was achieved. Monolayers of the pristine $\mathrm{Zn}_{2}$ (bim) 4 crystals with a thickness of $1 \mathrm{~nm}$ were obtained. The latter reported a direct bottom up synthesis, namely, freestanding MOF nanosheets were formed in the contact zone of a linker and a metal solution by using the diffusion-mediated modulation of the MOF growth kinetics [36]. As shown in Fig. 4(b), CuBDC lamellae with lateral dimensions of $0.5-4 \mu \mathrm{m}$ and thickness in the range 5-25 nm were synthesis by the three layer approach.

The breakthroughs in the synthesis of MOF nanosheets laid the foundations for MOF-based MSN membranes. $\mathrm{Zn}_{2}(\mathrm{bim})_{4}$ MSN membranes were assembled from a suspension of $\mathrm{Zn}_{2}$ (bim) ${ }_{4}$ nanosheets by a novel hot drop coating method, 

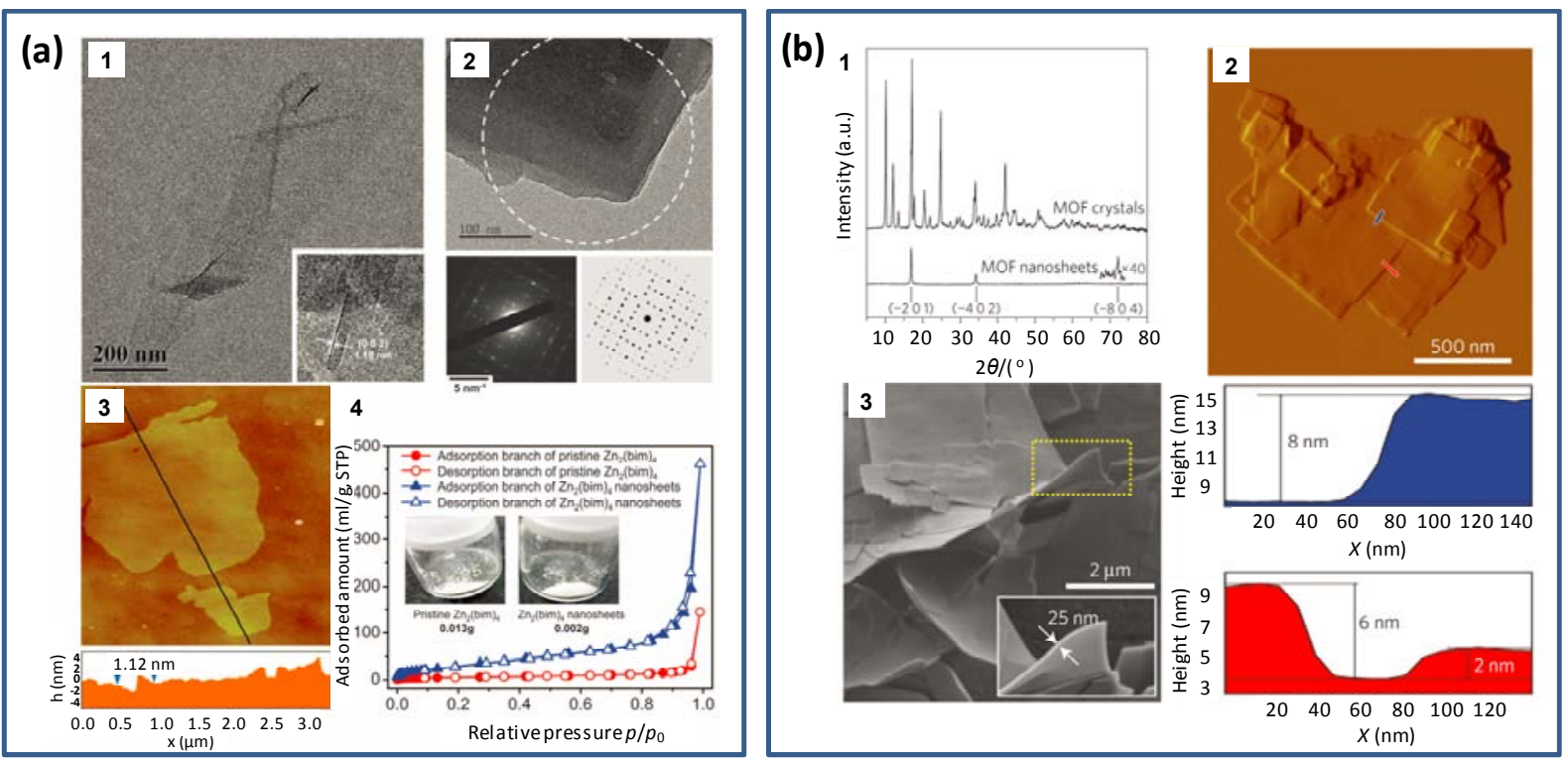

Fig. 4. (a) $\mathrm{Zn}_{2}$ (bim) ${ }_{4}$ nanosheets: (1) TEM image of a $\mathrm{Zn}_{2}(\text { bim })_{4}$ nanosheet; (2) SAED pattern shows the diffraction from (hk0) planes in a few-layer $\mathrm{Zn}_{2}$ (bim) $)_{4}$ nanosheet; (3) AFM topographical image of $\mathrm{Zn}_{2}(\mathrm{bim})_{4}$ nanosheets; (4) $\mathrm{N}_{2}$ adsorption-desorption isotherms (77 K) of the pristine $\mathrm{Zn}_{2}($ bim) 4 and $\mathrm{Zn}_{2}(\mathrm{bim})_{4}$ nanosheets. Adapted from Ref. [35]; (b) CuBDC nanosheets: (1) PXRD patterns for the bulk-type and nanosheet CuBDC; (2)AFM image and (3) SEM image of CuBDC nanosheets. Reproduced from Ref. [36] by permission of the Nature Publishing Group.

which showed $\mathrm{H}_{2}$ permeance of up to several thousand GPUs (gas permeation units, $1 \mathrm{GPU}=1 \times 10^{-6} \mathrm{~cm}^{3} / \mathrm{cm}^{2} \cdot \mathrm{sec} \cdot \mathrm{cmHg}$ at STP) with a $\mathrm{H}_{2} / \mathrm{CO}_{2}$ selectivity higher than 200 [35]. CuBDC mixed matrix membranes (MMMs) were prepared by incorporating $\mathrm{CuBDC}$ nanosheets within a polyimide matrix by the doctor-blading method [36]. In both cases, a significant relationship between the stacking status of the MOF nanosheets in the membranes and separating performance of the MSN membrane was identified. For the $\mathrm{Zn}_{2}$ (bim) ${ }_{4}$ MSN membranes, unlike conventional membranes, there is little or no association between $\mathrm{H}_{2}$ permeance and membrane thickness. In addition, an anomalous proportional relationship between permeance and selectivity was found (Fig. 5(a)). These abnormal findings can be explained by the order of the nanosheet stacking, i.e., the lamellar ordering of the nanosheets block the permeation pathway for $\mathrm{H}_{2}$ but have only a small effect on $\mathrm{CO}_{2}$ leakage (Fig. 5(a)). It can be speculated that an ultrapermeable and superselective molecular sieve membrane can be obtained with a membrane consisting of fully disordered $\mathrm{Zn}_{2}$ (bim) 4 MSNs.

For the CuBDC MMMs, the different MOF crystal morphology is crucial for the occupation of the gas permeation pathway by the molecular sieve and the filler-polymer integration. The
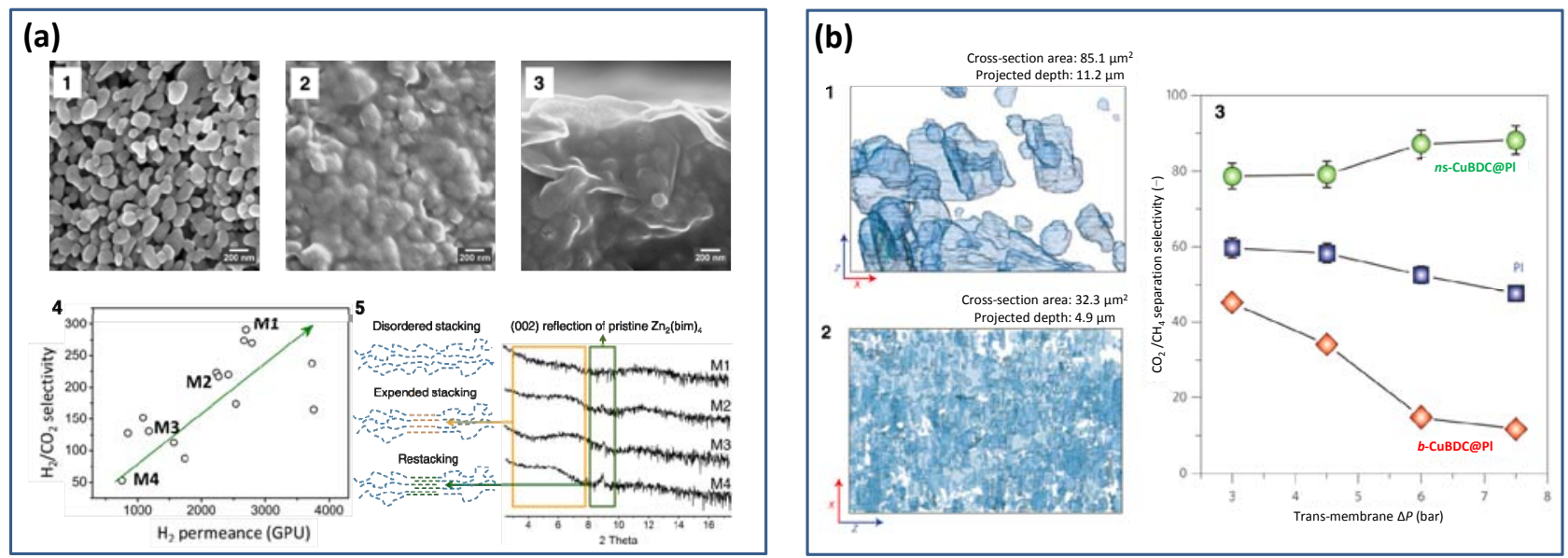

Fig. 5. (a) $\mathrm{Zn}_{2}$ (bim) $)_{4} \mathrm{MSN}$ membranes: SEM image of (1) bare porous a- $\mathrm{Al}_{2} \mathrm{O}_{3}$ support, (2) top view and (3) cross-sectional view of a $\mathrm{Zn} 2$ (bim) nanosheet layer on $\mathrm{a}-\mathrm{Al}_{2} \mathrm{O}_{3}$ support; (4) anomalous proportional relationship between selectivity and permeance measured from 15 membranes; (5) PXRD patterns of four membranes with different separation properties showing the importance of the stacking of the nanosheets on membrane performance.Adapted from Ref. [35]; (b) cross-sectional occupation of CuBDC in polyimide MMMs: (1) bulk-type and (2) nanosheet $\mathrm{CuBDC}_{\text {; }}(3) \mathrm{CO}_{2} / \mathrm{CH}_{4}$ separation selectivity as a function of the pressure difference across the membrane for MMMs containing bulk-type CuBDC and one containing CuBDC nanosheets, as well as a neat polyimide membrane. Reproduced from Ref. [36] by permission of the Nature Publishing Group. 
effective cross-sectional occupation of the CuBDC nanosheets was almost three times higher than that of bulk-type CuBDC crystals used as fillers at the same loading, resulting in a higher separation selectivity (Fig. 5(b)). Moreover, the selectivity of CuBDC MSN membrane increased slightly with upstream pressure, which was opposite to the general observation with both polymeric and conventional MOF MMMs (Fig. 5(b)).

\section{Conclusion and perspective}

In conclusion, the emergence of MOF materials brings new vitality to molecular sieve membrane research. Synthetic techniques developed and verified for zeolite membranes are readily transferable to the MOF membranes, leading to the evolution from purely inorganic materials to organic-inorganic hybrids [37]. Unlike traditional inorganic zeolites with a "rigid" framework, the structural flexibility of MOFs is prominent, which weakens the size-exclusion separation ability of MOF-based molecular sieve membranes. On the other hand, this dynamic structural behaviour is beneficial for adsorption-based separation, e.g., pevaporation. A large number of layered MOFs have been reported, and these MOFs can serve as a diverse source of MSNs when they are exfoliated to nm-scale thickness or controllably synthesized as freestanding high aspect ratio nanosheets. Recent breakthroughs in these aspects have opened the door to the rational design and synthesis of a new generation of molecular sieve membranes based on MOF nanosheets, leading to a remarkable improvement in separation performance by the reduction from $3 \mathrm{D}$ to $2 \mathrm{D}$.

We predict that the recent advances in the preparation and characterization of 2D layered MOFs will provide a platform for application-oriented fabrication of high performance membranes and pave the way for a breakthrough in membrane technology. Delaminated 2D MOFs can easily find application as mixed matrix membranes since no change in the polymeric membrane processing is needed, such as hollow fibre spinning and foil casting. In addition, the modifiable functionalities and dimensions of MOF materials can endow MOF-based membranes and films with a wide range of applications from catalytic membrane reactors (like zeolites, MOFs can act as heterogeneous catalysts, especially in fine chemical synthesis.) [38-40] to heating pumps [41], and from novel solar cells [42] to advanced LEDs [43]. Before the commercial implementation of MOF membranes, some important issues still need to be addressed such as their thermal and chemical stability [44], permeation and separation mechanism $[45,46]$ and scale-up preparation of MOF membranes at an acceptable cost [47].

\section{Acknowledgments}

This work was supported by the National Natural Science Fundation of China (21176231, 21276249, 21361130018).

Yanshuo Li

Dalian Institute of Chemical Physics, Chinese Academy of Sciences, Dalian 116023, Liaoning, China

Tel: +86-411-84379137
Fax: +86-411-84694447

E-mail: leeys@dicp.ac.cn

Received 27 February 2015

Published 20 May 2015

DOI: $10.1016 /$ S1872-2067(15)60838-5

\section{References}

[1] Robeson L M. J Membr Sci, 2008, 320: 390

[2] Lai Z P, Bonilla G, Diaz I, Nery J G, Sujaoti K, Amat M A, Kokkoli E, Terasaki O, Thompson R W, Tsapatsis M, Vlachos D G. Science, 2003, 300: 456

[3] Caro J, Noack M, Kölsch P. Microporous Mesoporous Mater, 2005, 11: 215

[4] Ismail A F, David L I B. J Membr Sci, 2001, 193: 1

[5] Li Y S, Chen H L, Liu J, Yang W S.J Membr Sci, 2006, 277: 230

[6] Zhou H, Li Y S, Zhu G Q, Liu J, Yang W S. Sep Purif Technol, 2009, 65: 164

[7] Morigami Y, Kondo M, Abe J, Kita H, Okamoto K. Sep Purif Technol, 2001, 25:251

[8] Li Y S, Liu J, Yang W S. J Membr Sci, 2006, 281: 646

[9] Li Y S, Yang W S. J Membr Sci, 2008, 316: 3

[10] Zhu G Q, Li Y S, Chen H L, Liu J, Yang W S. J Mater Sci, 2008, 43: 3279

[11] Li Y S, Liu J, Chen H L, Yang W S. Chin J Catal (李砚硕, 刘杰, 陈红 亮, 杨维慎, 林励吾. 催化学报), 2006, 27: 544

[12] Kitagawa S, Kitaura R, Noro S. Angew Chem Int Ed, 2004, 43: 2334.

[13] Yaghi O M, O’Keeffe M, Ockwig N W, Chae H K, Eddaoudi M, Kim J. Nature, 2003, 423: 705.

[14] Feréy G. Chem Soc Rev, 2008, 37: 191.

[15] Bux H, Liang F Y, Li Y S, Cravillon J, Wiebcke M, Caro J. J Am Chem Soc, 2009, 131: 16000

[16] Li Y S, Liang F Y, Bux H, Feldhoff A, Yang W S, Caro J. Angew Chem Int $E d, 2010,49: 548$

[17] Li Y S, Bux H, Feldhoff A, Li G L, Yang W S, Caro J. Adv Mater, 2010, 22: 3322

[18] Bux H, Feldhoff A, Cravillon J, Wiebcke M, Li Y S, Caro J. Chem Mater, 2011, 23: 2262

[19] Liu Y Y, Ng Z, Khan E A, Jeong H K, Ching C B, Lai Z P. Microporous Mesoporous Mater, 2009, 118: 296

[20] Zhang F, Zou X Q, Gao X, Fan S J, Sun F X, Ren H, Zhu G S. Adv Funct Mater, 2012, 22: 3583

[21] Yin H M, Wang J Q, Xie Z, Yang J H, Bai J, Lu J M, Zhang Y, Yin D H, Lin J Y. Chem Commun, 2014, 50: 3699

[22] Thornton A W, Dubbeldam D, Liu M S, Ladewig B P, Hill A J, Hill M R. Energy Environ Sci, 2012, 5: 7637

[23] Ryder M R, Civalleri B, Bennett T D, Henke S, Rudić S, Cinque G, Alonso F F, Tan J C. Phys Rev Lett, 2014, 113: 215502

[24] Liu X L, Li Y S, Zhu G Q, Ban Y J, Xu L Y, Yang W S. Angew Chem Int Ed, 2011, 50: 10636

[25] Kim W, Nair S. Chem Eng Sci, 2013, 104: 908

[26] Ng E P, Chateigner D, Bein T, Valtchev V, Mintova S. Science, 2012, 335: 70

[27] Jeong H K, Nair S, Vogt T, Dickinson L C, Tsapatsis M. Nature Mater, 2003, 2: 53

[28] Varoon K, Zhang X Y, Elyassi B, Brewer D D, Gettel M, Kumar S, Lee J A, Maheshwari S, Mitta A, Sung C Y, Cococcioni M, Francis L F, McCormick A V, Mkhoyan K A, Tsapatsis M. Science, 2011, 334: 72

[29] Agrawal K V, Topuz B, Jiang Z Y, Nguenkam K, Elyassi B, Francis L F, Tsapatsis M, Navarro M. AIChE J, 2013, 59: 3458

[30] Roth W J, Nachtigall P, Morris R E, Čejka J. Chem Rev, 2014, 114: 


\section{Graphical Abstract}

Chin. J. Catal., 2015, 36: 692-697 doi: 10.1016/S1872-2067(15)60838-5

\section{Molecular sieve membranes: From 3D zeolites to 2D MOFs}

Yanshuo Li*, Weishen Yang

Dalian Institute of Chemical Physics, Chinese Academy of Sciences

Remarkable improvement in separation performance can be achieved with 2D instead of 3D MOF membranes, opening the door to the rational design and synthesis of a new generation of molecular sieve membranes based on MOF nanosheets.

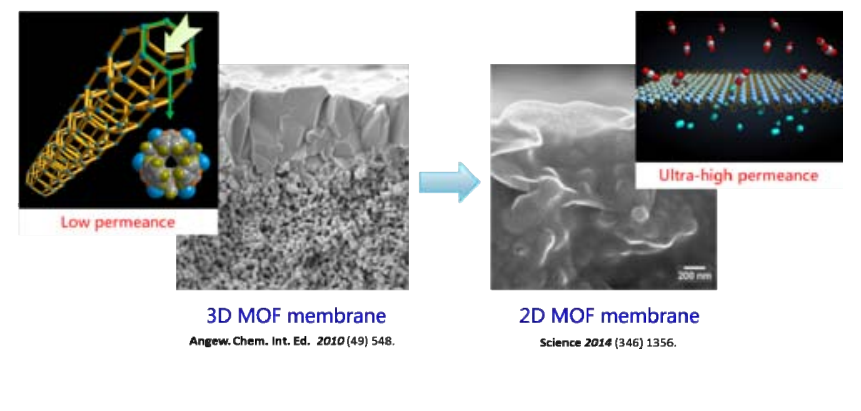

4807

[31] Cheetham A K, Rao C N R, Feller R K. Chem Commun, 2006: 4780

[32] Li P Z, Maeda Y, Xu Q. Chem Commun, 2011, 47:8436

[33] Gallego A, Hermosa C, Castillo O, Berlanga I, Gómez-García C J, Mateo-Martí E, Martínez J I, Flores F, Gómez-Navarro C, Gómez-Herrero J, Delgado S, Zamora F. Adv Mater, 2013, 25: 2141

[34] Tan J C, Saines P J, Bithell E G, Cheetham A K. ACS Nano, 2012, 6: 615

[35] Peng Y, Li Y S, Ban Y J, Jin H, Jiao W M, Liu X L, Yang W S. Science, 2014, 346: 1356

[36] Rodenas T, Luz I, Prieto G, Seoane B, Miro H, Corma A, Kapteijn F, Llabres i Xamena F X, Gascon J. Nature Mater, 2015, 14: 48

[37] Caro J. Curr Opin Chem Eng, 2011, 1:77

[38] Lee J Y, Farha O K, Roberts J, Scheidt K A, Nguyen S B T, Hupp J T. Chem Soc Rev, 2009, 38: 1450
[39] Nguyen L T L, Le K K A, Phan, N T S. Chin J Catal (催化学报), 2012, 33: 688

[40] Yilmaz B, Trukhan N, Muller U. Chin J Catal (催化学报), 2012, 33: 3

[41] Henninger S K, Habib H A, Janiak C. J Am Chem Soc, 2009, 131: 2776

[42] Li Y F, Pang A Y, Wang C J, Wei M D. J Mater Chem, 2011, 21: 17259

[43] Wang M S, Guo S P, Li Y, Cai L Z, Zou J P, Xu G, Zhou W W, Zheng F K, Guo G C. J Am Chem Soc, 2009, 131: 13572

[44] Liu X L, Li Y S, Ban Y J, Peng Y, Jin H, Bux H, Xu L Y, Caro J, Yang W S. Chem Commun, 2013, 49: 9140

[45] Keskin S, Liu J C, Johnson J K, Sholl D S. Micropor Mesopor Mater, 2009, 125: 101

[46] Pera-Titus M. Chem Rev, 2014, 114: 1413

[47] Brown A J, Brunelli N A, Eum K, Rashidi F, Johnson J R, Koros W J, Jones C W, Nair S. Science, 2014, 345: 72 